\title{
Diagnosis of Pulmonary Embolism: Conventional Ventilation/Perfusion SPECT Is Superior to the Combination of Perfusion SPECT and Nonenhanced CT
}

\author{
Karin Palmowski ${ }^{a, c, d}$ Ute Oltmanns ${ }^{a, c}$ Michael Kreuter ${ }^{a, c}$ Felix M. Mottaghy ${ }^{e}$ \\ Moritz Palmowski ${ }^{\text {b, e }}$ Florian F. Behrendt ${ }^{\mathrm{e}}$ \\ a Department of Pneumology and Respiratory Critical Care Medicine, Thoraxklinik Heidelberg, University of \\ Heidelberg, ${ }^{\mathrm{b}}$ Academic Radiology Baden-Baden, Diagnostic and Interventional Radiology, University Medical \\ Center Heidelberg, ' Member of the German Center for Lung Research (DZL), Translational Lung Research Center

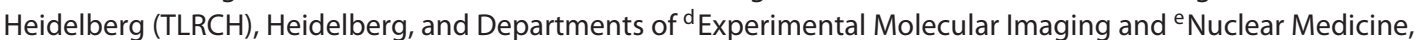 \\ RWTH-Aachen University, Aachen, Germany
}

\section{Key Words}

Pulmonary embolism · Single-emission photon CT .

Ventilation · Perfusion

\begin{abstract}
Background: Ventilation/perfusion single-emission photon CT (V/P-SPECT) is widely used to detect pulmonary embolism (PE). Any pathological deficit on P-SPECT with a corresponding unremarkable V-SPECT is considered an embolism. This means that a deficit on P-SPECT with a corresponding deficit on the ventilation scan correlates with other lung pathologies such as pneumonia, bullous emphysema or tumor. In principle, it is possible to identify any of these lung pathologies on nonenhanced chest $C T$ and so this technique has the potential to replace V-SPECT in the diagnosis of PE. Today, SPECT/CT hybrid imaging systems are increasingly applied in clinical routines. Objectives: We investigated whether embolism can be diagnosed using a combined P-SPECT/CT hybrid imaging ap-
\end{abstract}

proach without V-SPECT. Methods: Ninety-three patients with clinically suspected embolism were investigated with standard V/P-SPECT and a nonenhanced CT scan on a combined SPECT/CT system. A diagnosis of embolism was based on V/P-SPECT (gold standard). P-SPECT/CT datasets were blinded and analyzed without any knowledge of the V-SPECT data. The accuracy of P-SPECT/CT was compared to the gold standard. Results: Embolism was diagnosed in 24/93 patients using V/P-SPECT. In total, 57 lung lobes were affected. P-SPECT/CT significantly $(p<0.01)$ overdiagnosed embolism in nonaffected patients. In total, 36 cases with 88 affected lung lobes were shown. The sensitivity was $95.8 \%$, the specificity $82.6 \%$, the false-negative rate $4.2 \%$ and the false-positive rate $17.3 \%$. Conclusions: Our results demonstrate that a nonenhanced $\mathrm{CT}$ scan in a novel hybrid imaging system cannot replace V-SPECT in the scintigraphy-based diagnosis of PE. V-SPECT increases specificity and reduces the number of false-positive results when compared to 'perfusion-only' SPECT/CT.

(C) 2014 S. Karger AG, Base

\section{KARGER}

E-Mail karger@karger.com

www.karger.com/res
(C) 2014 S. Karger AG, Basel

$0025-7931 / 14 / 0884-0291 \$ 39.50 / 0$
Dr. Karin Palmowski, MD

Department of Pneumology and Respiratory Critical Care Medicine

Thoraxklinik Heidelberg, University of Heidelberg

DE-69120 Heidelberg (Germany)

E-Mail karin.palmowski@ @ed.uni-heidelberg.de 


\section{Introduction}

Acute pulmonary embolism (PE) is a severe and potentially fatal disease, with a mortality rate of approximately $30 \%$ if left untreated [1]. Diagnosis remains a challenge despite the many guidelines and scoring systems available.

Apart from clinical probability scores and other diagnostic procedures, there are two imaging modalities that are predominantly used to determine PE. Contrast-enhanced CT is the main investigational method for the diagnosis of PE in Germany (interdisciplinary S2 guidelines [2]) as well as in many other countries [3-5]. However, for patients with renal dysfunction, a critical illness or a contrast-medium allergy, applying ventilation/perfusion $(\mathrm{V} / \mathrm{P})$ scintigraphy is preferable as there are no contraindications and no reports of allergic reactions [6].

If pulmonary perfusion scintigraphy reveals a conspicuous hollow of tracer uptake, which correlates with a deficit on ventilation scintigraphy ('match-finding'), PE can be ruled out. However, if there is a notch on perfusion scintigraphy and the ventilation in the corresponding lobe/segment is unremarkable ('mismatch-finding'), PE must be suspected.

In the past, planar 2-dimensional V/P scintigraphy was applied. Today, single-emission photon CT (SPECT) has replaced planar imaging, providing better sensitivity and/or specificity [7-11], not least as a result of the new interpretation criteria. V/P-SPECT represents a transition from planar scintigraphic to cross-sectional imaging, with data acquired tomographically and analyzed as a 3-dimensional dataset [12-14]. A comprehensive study by Bajc et al. [15] showed the high diagnostic sensitivity (99\%) and specificity (93\%) of V/P-SPECT, underlining the reliability of this method.

Hybrid SPECT/CT devices have recently been introduced; these potentially improve diagnostic accuracy by enabling an anatomical characterization of scintigraphic abnormalities. Non-contrast-enhanced thoracic CT imaging may indicate causes (other than PE) for abnormal lung perfusion by demonstrating morphological abnormalities such as pneumonia, bullous emphysema or tumor. Consequently, combined P-SPECT/CT scanning may replace $\mathrm{V} / \mathrm{P}-\mathrm{SPECT}$ in the future. This is of great interest, particularly with regard to patients who cannot undergo ventilation imaging due to clinical instability, massive dyspnea, incompliance or reduced consciousness. In addition, the examination time is cut by approximately half.
We investigated the diagnostic accuracy of combined $\mathrm{P}$-SPECT/CT imaging in the diagnosis of $\mathrm{PE}$ and compared it to conventional V/P-SPECT.

\section{Material and Methods}

\section{Patients}

From December 2011 to October 2012, a total of 93 patients (55 men and 38 women with a mean age of $70 \pm 14$ years) underwent pulmonary V/P-SPECT and subsequent non-contrast-enhanced thoracic CT imaging on a hybrid SPECT/CT scanner for clinically suspected PE. The majority of patients had contraindications to the use of iodine-containing contrast media including renal dysfunction or allergy. All patients were enrolled in this study retrospectively, so permission from the institutional review board was not required.

\section{Pulmonary SPECT/CT}

Pulmonary SPECT included a P-SPECT and V-SPECT study on patients with their arms raised. SPECT/CT was performed on a Symbia TruePoint SPECT/CT T16 (Siemens Healthcare USA, Inc., Malvern, Pa., USA). In SPECT mode, 64 projections (matrix: $128 \times 128$ ) were acquired in one bed position on a $360^{\circ}$ trajectory. In each position, detector events within one energy window were collected during $24 \mathrm{~s}$ for the ventilation scan and $8 \mathrm{~s}$ for the perfusion scan (window center/width in $\mathrm{keV} / \%$ : 140/15). Reconstruction of spatial activity distribution (voxel cube edge length: $4.8 \mathrm{~mm}$ ) was carried out using the Flash3D ${ }^{\mathrm{TM}}$ algorithm on a Siemens syngo MI Workplace. CT-based attenuation correction was applied during the iterative reconstruction (4 iterations/8 subsets).

Ventilation scans were done after inhalation of $400 \mathrm{MBq}$ ${ }^{99} \mathrm{~m} \mathrm{Tc}$-technegas over 3-5 respiratory cycles prepared in a generator (Technegas Generator, Tetley Manufacturing Ltd, Sydney, Australia). Immediately after the ventilation scintigraphy, perfusion studies were performed after intravenous injection of $180 \mathrm{MBq}$ of ${ }^{99 m} \mathrm{Tc}$-macroaggregated albumin (Mallinckrodt Inc., Hazelwood, Mo., USA) administered over 5 respiratory cycles. Prior to these two applications, patients received an inhalatory bronchodilatator and were instructed to perform several deep inspirations for optimal recruitment by the lung capillaries. CT parameters for the low-dose nonenhanced CT were: collimation $16 \times 1.2 \mathrm{~mm}$, pitch 1.0 , rotation time $0.6 \mathrm{~s}$, effective tube current-time product of $25 \mathrm{mAs}$ and tube voltage of 110 $\mathrm{kV}$. CT image reconstruction was performed with a mediumsmooth soft-tissue kernel (window center $50 \mathrm{HU}$; window width $400 \mathrm{HU}$ ) and a lung-kernel (window center $600 \mathrm{HU}$; window width $1,200 \mathrm{HU}$ ) at a slice thickness of $5 \mathrm{~mm}$ and increment of $5 \mathrm{~mm}$.

\section{Image Analysis}

Analysis of the acquired images was performed by two reviewers (both were radiologists and nuclear medicine physicians with 7 and 8 years of experience in the field of chest imaging, respectively) blinded to the clinical results. Diagnosis of a PE was done in a consensus reading.

Gold Standard. In a first step, V/P-SPECT images were analyzed. According to clinical standards, the criterion for the diagno- 
Fig. 1. True-positive finding: bilateral lung embolism. a, b The standard technique of V/P-SPECT for the diagnosis of PE demonstrates nonperfused (arrows) but ventilated parts of the right and left lung (mismatch), pointing to the diagnosis of bilateral lung embolisms. c, d The proposed technique of P-SPECT combined with a nonenhanced CT scan excludes a morphological reason (e.g. tumor, pneumonia or emphysema) for the nonperfused parts of the lung. Consequently, the perfusion defect finding has to be interpreted as a PE.

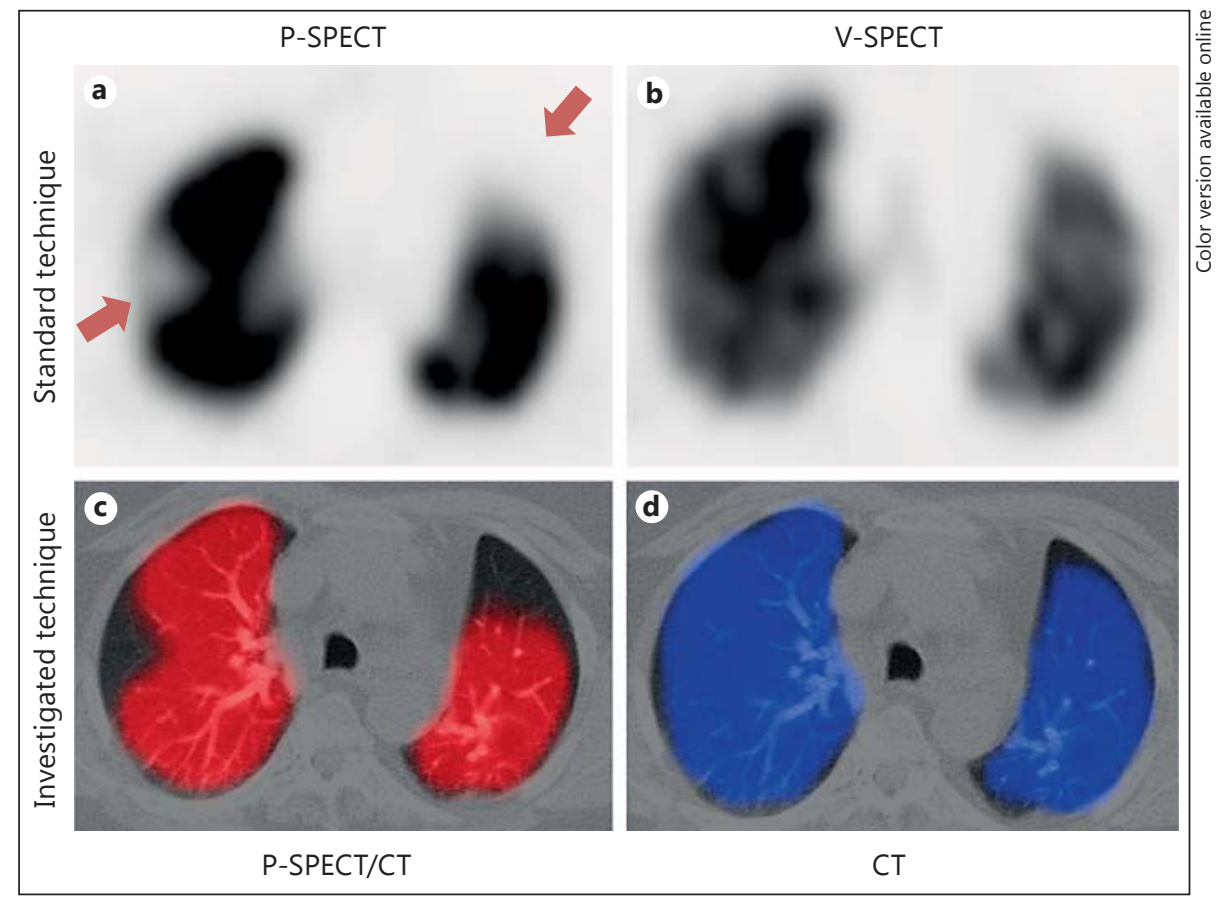

Table 1. Accuracy of P-SPECT/CT compared to the gold standard (V/P-SPECT)

\begin{tabular}{lll}
\hline & PE findings $(\mathrm{n}=24)$ & No PE found $(\mathrm{n}=69)$ \\
\hline P-SPECT/CT indicates PE & 23 true-positive & 12 false-positive \\
P-SPECT/CT rules out PE & 1 false-negative & 57 true-negative \\
\hline
\end{tabular}

sis of PE was a notch in a lung lobe on P-SPECT that corresponded to an unremarkable V-SPECT. The diagnosis of PE and the number of affected lung lobes were recorded.

$P$-SPECT and CT. Four weeks later, the reviewers analyzed the P-SPECT images again in conjunction with the respective nonenhanced CT images. The order of cases $(n=93)$ was chosen at random to avoid any bias. Diagnostic criteria for PE included the combined findings of a perfusion defect on P-SPECT and a corresponding unremarkable chest CT scan. Radiological findings, defined in order to rule out lung embolism, were of morphologically depictable nonventilated lung tissue (e.g. bullae, strong emphysema, lung tumors and consolidated lung tissue).

\section{Statistical Analysis}

Diagnostic performance was calculated as sensitivity, specificity and false-positive and false-negative values. The two-sided nonparametric paired Wilcoxon test was used to compare the results from the gold standard (V/P-SPECT) and the novel P-SPECT/ CT scan. All statistical analyses were conducted using SPSS 19 (IBM, Somers, Brewster, N.Y., USA).

Nonenhanced CT and P-SPECT versus $\mathrm{V} / \mathrm{P}-\mathrm{SPECT}$ for PE Diagnosis

\section{Results}

A diagnosis of PE was confirmed by V/P-SPECT in 24 patients, with a total of 57 affected lung lobes. PE was excluded in the remaining 69 patients.

The analysis of the P-SPECT images in combination with the CT images showed a sensitivity of $95.8 \%$ (table 1; fig. 1) for the diagnosis of PE. Only one episode of PE was not detected, resulting in a false-negative rate of $4.2 \%$. However, the specificity of P-SPECT/CT was comparatively low at $82.6 \%$ and the rate of false-positives was high at $17.3 \%$ (table 1). Overall, using P-SPECT in combination with $\mathrm{CT}$ imaging, $\mathrm{PE}$ was identified in 88 pulmonary lobes from a total of 36 patients. The differences between V/P-SPECT and P-SPECT/CT were significant $(\mathrm{p}<0.01)$.

Analysis of all true-negative cases in the P-SPECT/CT showed that causes for the pathological absence of perfusion, such as lung tumors, pneumonia or bullous emphysema, were detected reliably by CT scan (fig. 2-4). 
Fig. 2. True-negative finding: pneumonia and dystelectasis. a, b The standard technique V/P-SPECT demonstrates nonperfused and nonventilated areas in both sides of the lung (arrows). Due to the matchfinding, PE can be excluded as a reason for the perfusion defect. c, d The proposed technique P-SPECT/CT demonstrates a morphological reason for the perfusion defect (pneumonia and dystelectasis), so PE can be excluded here.

Fig. 3. True-negative finding: bullous emphysema. a, b The standard technique V/PSPECT demonstrates a nonperfused and nonventilated area in the right ventral part of the lung. Due to the match-finding, PE can be excluded as a reason for the perfusion defect. c, d The proposed technique P-SPECT/CT demonstrates a morphological reason for the perfusion defect (bullous emphysema), so PE can be ruled out.
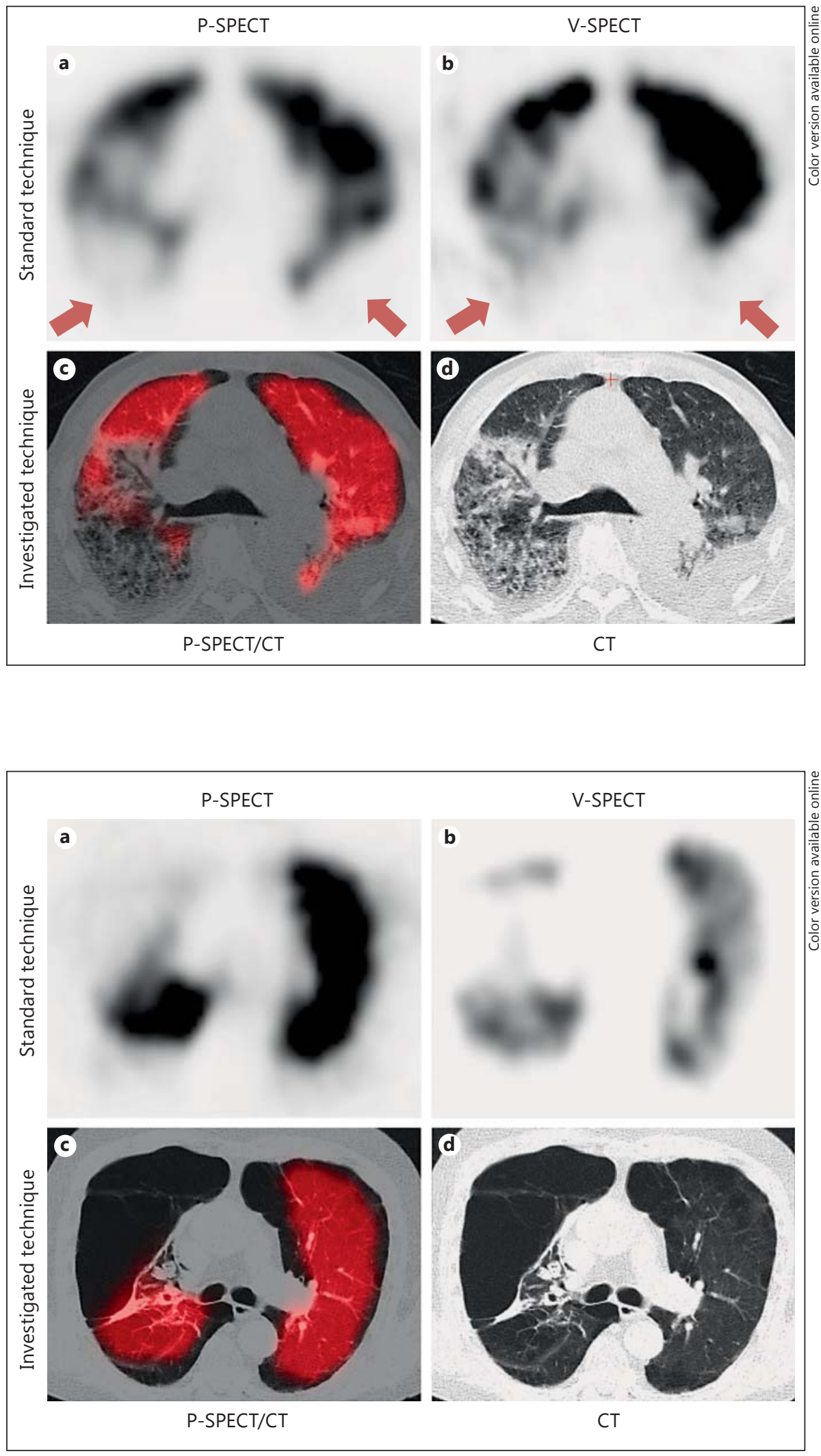
Fig. 4. True-negative finding: lung tumor. a, b The standard technique V/P-SPECT demonstrates a small nonperfused and nonventilated area within the right lower lobe (arrows). The match-finding excludes $\mathrm{PE}$ as a cause for the small perfusion defect. c, $\mathbf{d}$ The proposed technique $\mathrm{P}$-SPECT/CT demonstrates a morphological correlation for the perfusion defect (lung tumor), and therefore rules out PE.

Fig. 5. False-positive finding: misdiagnosis of PE by P-SPECT/CT. a, b The standard technique V/P-SPECT demonstrates nonperfused and nonventilated areas in both sides of the lungs (arrows). Due to the match-finding, PE can be excluded for the perfusion defect. c, d The proposed technique P-SPECT/CT excludes a morphological reason for the perfusion defect, leading to a misdiagnosis of PE (i.e. a falsepositive result).
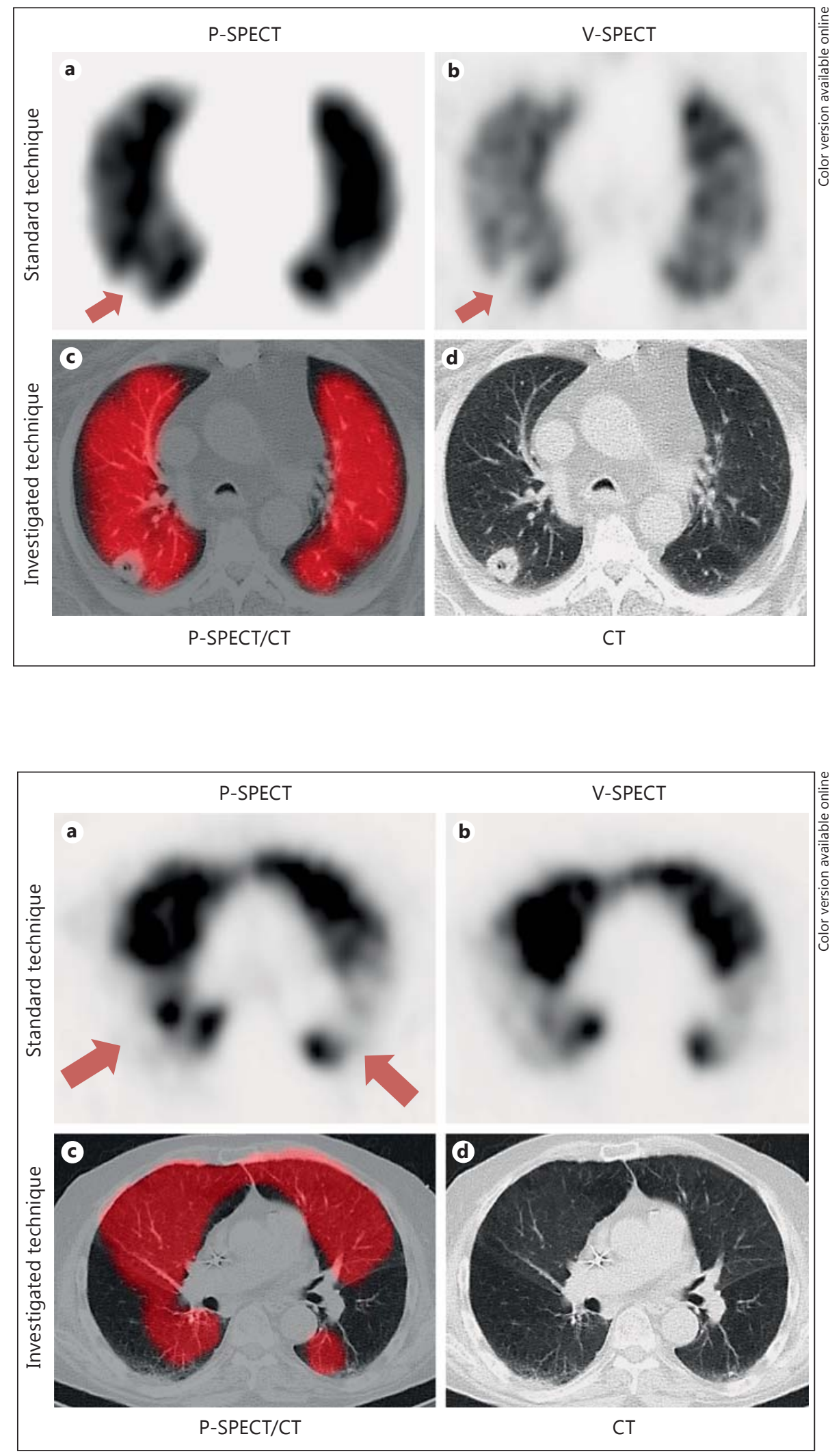
However, all 12 patients with a false-positive diagnosis of PE in the P-SPECT/CT had a corresponding lack of perfusion and ventilation in the V/P-SPECT which did not correlate with any morphological changes on CT imaging (fig. 5).

\section{Discussion}

Despite the many guidelines and clinical scoring systems available, PE remains an important, underdiagnosed condition that impacts profoundly on patient morbidity and mortality [1]. The all-cause mortality rate of patients with unsuspected $\mathrm{PE}$ is even higher than the mortality rate of patients with suspected PE [16]. V/PSPECT and contrast-enhanced CT of the chest are widely used as primary diagnostic methods [6] with sensitivities and specificities greater than 90-95\% [3]. However, only limited data are available on the comparison of diagnostic sensitivity and specificity of these two cross-sectional imaging techniques. Reinartz et al. [9] found that contrastenhanced multidetector CT had a specificity (98\%) superior to V/P-SPECT (91\%), but that V/P-SPECT had a better sensitivity (97\%) than multidetector CT $(86 \%)$. In a large study by Bajc et al. [15], V/P-SPECT was found to have a diagnostic sensitivity of $99 \%$ and a specificity of 93\%.

Ventilation scintigraphy as part of the V/P-SPECT is applied to rule out other possible causes for abnormal perfusion (e.g. pneumonia, bullous emphysema or tumor). In principle, this may also be achieved with a noncontrast-enhanced thoracic CT. Furthermore, an easily applicable non-contrast-enhanced CT scan might provide valuable information about associated thoracic pathologies.

Consequently, a combined P-SPECT/CT scan may replace standard V/P-SPECT. The aim of our study was to investigate whether ventilation scintigraphy can be replaced by non-contrast-enhanced CT. This is of great interest, particularly for patients who cannot undergo ventilation imaging due to clinical instability, massive dyspnea, incompliance or reduced consciousness. In addition, examination time is cut by approximately half. A prospective study published in 1996 [17] showed planar perfusion scintigraphy (without ventilation) to have a sensitivity of $86 \%$ and a specificity of $93 \%$ in comparison to the reference diagnostic standard (pulmonary angiography). We used V/P-SPECT as our reference standard due to its high diagnostic accuracy $[1,9,15,18,19]$. However, as a limiting factor, it is important to be aware that small emboli in the lingula and the right middle lobe may be missed by P-SPECT due to the shape of these segments. Our patients all tolerated V/P-SPECT and CT without complications. Most were unable to undergo contrast CT scanning due to renal dysfunction, critical illness or contrast-medium allergy. As a result of this, we had a selected study population. Our gold standard, V/PSPECT, identified PE in $25.8 \%$ of our patients (24/93). However, when analyzing the novel method, P-SPECT/ CT, PE was found in 38.7\% (36/93). Analyzing the images of the 12 patients with a false-positive result, PSPECT demonstrated a notch within a lung lobe without corresponding anatomical abnormalities on the CT image (e.g. pneumonia, bullae or emphysema). Altogether, this represents a false-positive rate of $17.3 \%$ with the proposed method. Recently, Gutte et al. [1] reported the detection of PE with combined V/P-SPECT and low-dose $\mathrm{CT}$ in comparison to multidetector CT angiography. They showed that P-SPECT combined with low-dose CT had a sensitivity of $93 \%$, but a low specificity of $51 \%$. The sensitivity was similar to our finding of $95.8 \%$, but the specificity in our study was higher at $82.6 \%$. We found no obvious explanation for this discrepancy, but differences in the study populations and algorithms for final diagnosis may have been contributing factors. Our results show that the sensitivity of P-SPECT/CT is comparable to V/PSPECT because it was based on the same perfusion scintigraphy in both cases.

Hospitalized patients often suffer from comorbidities, many of which are associated with hypoxia. These include fever, anemia, pain, cardiovascular diseases (e.g. septum dysplasia and pulmonary hypertension), neurological deficits (e.g. paralysis of respiratory muscles), central brain diseases and PE. It is known that hypoxia induces vasoconstriction (the Euler-Liljestrand mechanism) [20]. Potentially, this hypoxia and the resulting vasoconstriction is caused by a mild functional ventilation disruption present on the V-SPECT but not morphologically visible on the low-dose CT. Furthermore, obstructive airway diseases, which influence the ventilation on SPECT imaging due to either severe narrowing or airway closure [21], may be the reason for a perfusion deficit (generated by the Euler-Liljestrand mechanism); as a consequence, PE could be mimicked in perfusion scintigraphy.

This hypothesis would explain a defect in the P-SPECT, even though the corresponding CT revealed no structural changes. The consequence is a mismatch between $\mathrm{P}$ SPECT and CT which is interpreted falsely as a PE, representing a false-positive finding. It remains unclear, 
however, why some patients show a notch within a lung lobe on the pulmonary P-SPECT without any morphological correlate in the corresponding lung section on CT imaging.

A limitation of all available studies including ours is the lack of an independent gold standard with a sensitivity and specificity of $100 \%$. All imaging procedures produce false-positive and false-negative results. A further limitation of our study is that the comorbidities and the final clinical diagnosis given by the physician were not recorded. However, this was not the aim of our investigation. We wanted to prove the advantages and disadvantages of P-SPECT in combination with a non-contrastenhanced CT scan in a modern hybrid SPECT/CT system in comparison to $\mathrm{V} / \mathrm{P}-\mathrm{SPECT}$ with its high diagnostic accuracy. By matching these imaging methods, a significant difference between these two procedures could be demonstrated.
The two methods were comparable in sensitivity, which was based on the perfusion scintigraphy. Particularly with regard to patients who cannot perform ventilation imaging, such as those who are clinically unstable or unconscious, this presents an option to identify or exclude PE. In all other patients, it is considered mandatory to perform perfusion and ventilation scintigraphy because perfusion-only SPECT/CT is accompanied by reduced specificity and more false-positive results.

\section{Conclusion}

Our results suggest that ventilation scintigraphy should still be a fixed part of SPECT/CT imaging for the diagnosis of PE. It increases the specificity and reduces the number of false-positive results when compared to perfusion-only SPECT/CT.

\section{References}

1 Gutte H, Mortensen J, Jensen CV, et al: Detection of pulmonary embolism with combined ventilation-perfusion SPECT and low-dose CT: head-to-head comparison with multidetector CT angiography. J Nucl Med 2009;50: 1987-1992.

2 Interdisziplinäre S2-Leitlinie: Diagnostik und Therapie der Bein- und Beckenvenenthrombose und der Lungenembolie. VASA 2010;39: $1-39$.

- 3 Stein PD, Fowler SE, Goodman LR, et al: Multidetector computed tomography for acute pulmonary embolism. N Engl J Med 2006; 354:2317-2327.

4 Stein PD, Woodard PK, Weg JG, et al: Diagnostic pathways in acute pulmonary embolism: recommendations of the PIOPED II investigators. Radiology 2007;242:15-21.

$\checkmark 5$ Strashun AM: A reduced role of V/Q scintigraphy in the diagnosis of acute pulmonary embolism. J Nucl Med 2007;48:1405-1407.

6 Hofman MS, Beauregard JM, Barber TW, et al: 68Ga PET/CT ventilation-perfusion imaging for pulmonary embolism: a pilot study with comparison to conventional scintigraphy. J Nucl Med 2011;52:1513-1519.

-7 Bajc M, Olsson CG, Olsson B, Palmer J, Jonson B: Diagnostic evaluation of planar and tomographic ventilation/perfusion lung images in patients with suspected pulmonary emboli. Clin Physiol Funct Imaging 2004;24:249-256.
8 Reinartz P, Schirp U, Zimny M, et al: Optimizing ventilation-perfusion lung scintigraphy: parting with planar imaging. Nucl Med 2001;40:38-43.

-9 Reinartz P, Wildberger JE, Schaefer W, et al: Tomographic imaging in the diagnosis of pulmonary embolism: a comparison between V/Q lung scintigraphy in SPECT technique and multislice spiral CT. J Nucl Med 2004;45: 1501-1508.

10 Collart JP, Roelants V, Vanpee D, et al: Is a lung perfusion scan obtained by using single photon emission computed tomography able to improve the radionuclide diagnosis of pulmonary embolism? Nucl Med Commun 2002;23:1107-1113.

-11 Palmer J, Bitzén U, Jonson B, Bajc M: Comprehensive ventilation/perfusion SPECT. J Nucl Med 2001;42:1288-1294.

12 Ling IT, Naqvi HA, Siew TK, Loh NK, Ryan GF: SPECT ventilation perfusion scanning with the addition of low-dose CT for the investigation of suspected pulmonary embolism. Intern Med J 2012;42:1257-1260.

13 De Geeter FW: Tomographic imaging in the diagnosis of pulmonary embolism: still, we do not know. J Nucl Med 2005;46:2119-2120.

14 Meignan MA: Lung ventilation/perfusion SPECT: the right technique for hard times. J Nucl Med 2002;43:648-651.
15 Bajc M, Olsson B, Palmer J, Jonson B: Ventilation/perfusion SPECT for diagnostics of pulmonary embolism in clinical practice. J Intern Med 2008;264:379-387.

16 King GG: Tomographic imaging of small airways. Respiration 2012;84:265-274.

17 Miniati M, Pistolesi M, Marini C, et al: Value of perfusion lung scan in the diagnosis of pulmonary embolism. Results of the Prospective Investigative Study of Acute Pulmonary Embolism Diagnosis (PISA-PED). Am J Respir Crit Care Med 1996;154:1387-1393.

18 Bajc M, Bitzén U, Olsson B, et al: Lung ventilation/perfusion SPECT in the artificially embolized pig. J Nucl Med 2002;43:640-647.

19 Gutte H, Mortensen J, Jensen C, et al: Added value of combined simultaneous lung ventilation-perfusion single-photon emission computed tomography/multislice-computed tomography angiography in two patients suspected of having acute pulmonary embolism. Clin Respir J 2008;1:52-55.

20 Schmidt RF, Thews G: Lungenatmung: Lungenperfusion und Arterialisierung des Blutes, vol 27; in Physiologie des Menschen. Berlin, Springer, 1997, pp 587-588.

21 Shteinberg M, Segal-Trabelsy M, Adir Y, et al: Clinical characteristics and outcomes of patients with clinically unsuspected pulmonary embolism versus patients with clinically suspected pulmonary embolism. Respiration 2012;84:492-500.
Nonenhanced CT and P-SPECT versus V/P-SPECT for PE Diagnosis 